\title{
THE RADIOLOGICAL AND BACTERIOLOGICAL ASSESSMENT OF TUBERCULOUS LUNG LESIONS REMAINING AFTER CHEMOTHERAPY
}

\author{
BY \\ A. W. T. EADE,* G. KENT HARRISON, S. E. LARGE, $\dagger$ J. MACKAY-DICK, \\ LYNNE REID, AND R. W. RIDDELL \\ From the Connaught Hospital (Army Chest Centre), Hindhead, Surrey, and the Institute of \\ Diseases of the Chest, Brompton Hospital, London
}

(RECEIVED FOR PUBLICATION JANUARY 20, 1959)

Bacteriological study of residual lesions resected from patients who have received chemotherapy for pulmonary tuberculosis offers a means of evaluating the efficacy of such therapy and may also give some guidance concerning the place of partial lung resection in the treatment of pulmonary tuberculosis.

In recent years there have been a number of such studies (d'Esopo, Bernstein, Decker, Raleigh, and Steenken, 1953; Auerbach, Hobby, Small, Lenert, and Comer, 1955 ; Stewart, Turnbull, and Macgregor, 1956; Todd, Teare, and Gordon, 1956; Bottrill and Edge, 1957; Hurford and Valentine, 1957 ; Cotter, Foreman, and Seal, 1958), but the results of bacteriological examination have not always been related to the particular drug régime employed or to the type of disease present. Furthermore, the specimens studied have often been derived from patients in whom resection has been undertaken because treatment by chemotherapy had failed or was considered inadequate as a definitive form of treatment. For these reasons conclusions from such studies have been limited.

The group of patients studied in this investigation differs from those previously reported in several important respects. First, they have all received the same régime of chemotherapy pre-operatively; secondly, the entire period of pre-operative treatment (10 to 25 months) has been carried out in hospital under supervision; thirdly, the special circumstances of these patients in many cases necessitated resection of residual diseased areas that normally might not have demanded surgery. Finally, the extent and nature of the disease have been confirmed

\footnotetext{
*Present address: Bolingbroke Hospital, London, S.W.11.

tPresent address: B.M.H., Kinrara, c/o G.P.O., Kuala Lumpur. Malaya.
}

tomographically at several stages in all patients. $\breve{.}$ Moreover, the specimen studies reported here $\vec{\Phi}$ have been carried out independently at two $\vec{\bullet}$ laboratories. The results of these studies have been correlated with the bacteriological and radiological findings, and from the informationo so gained certain tentative conclusions have been made regarding both the efficacy of chemotherapy and the place of resectional surgery in the management of pulmonary tuberculosis.

\section{The Patients}

The patients were all Gurkha soldiers aged 20-40马 years serving with the Brigade of Gurkhas in the Far East Land Forces in Malaya, Hong Kong, and Singapore. They were all first found to be을 suffering from pulmonary tuberculosis between $\widetilde{x}$ 1955 and 1957, and without exception were윽 otherwise fit men.

Since 1951 all Gurkha soldiers suffering from tuberculosis have been treated at the British? Military Hospital, Kinrara, Malaya. Treatment? there rarely included surgical procedures since $>$ local facilities were limited. Before 1955 the usual practice was that after treatment patients were invalided to Nepal, where life is arduous and facilities for follow-up and continued chemo-O therapy are scanty. In these circumstances it wasc felt that as a general rule resection should be the treatment of choice for any patient who had moree than minimal residual disease after chemotherapy. Accordingly the Director General, Army Medical? Services, made arrangements for selected patients 0 to be sent to the United Kingdom for thoracico surgery at the Connaught Hospital (Army Chesto Centre). Since 1955 the Gurkha soldier suffering from pulmonary tuberculosis has been entitled tog 24 months' continuous hospital treatment on fulb pay. Consequently most of the patients? 
transferred to the United Kingdom to date have already received chemotherapy for more than one year, and some of those transferred initially had received drugs for two years.

It is the first group of 59 patients transferred for partial lung resection under this scheme that forms the subject of this investigation.

\section{Pre-operative Treatment and Management}

All patients were treated in hospital. They were kept in bed only until they were afebrile, clinically well, and the sputum was bacteriologically negative. Thereafter, the degree of physical activity allowed was regulated according to the individual case, but strict bed rest for two hours daily was compulsory for all patients. Sputum was examined on admission and thereafter at intervals of six weeks.

Initial radiological assessment included posteroanterior (P.A.) and lateral radiographs, and fullplate antero-posterior tomography in all cases; subsequently P.A. chest radiographs were taken at intervals of six weeks throughout treatment. Cavity closure, when it was considered that this had occurred, was confirmed by tomography.

The drug régime was uniform. Initially the patient received streptomycin, $1.0 \mathrm{~g}$. daily, and isoniazid, $100 \mathrm{mg}$. twice daily, for either 30 or 60 days depending upon the initial assessment of the case. Thereafter both drugs were given in the same dosage every other day, and this régime was continued until operation. Three patients received a longer period of daily chemotherapy initially (90 days for two, and 85 days for the other), but their treatment differed in no other way.

No additional treatment, e.g., artificial pneumothorax, pneumoperitoneum, was used. No patient had received previous treatment for tuberculosis.

\section{Case Assessment in the U.K.}

All previous radiographs and tomographs were re-examined by three independent observers, and each case was reassessed clinically, radiologically, and bacteriologically. Antero-posterior tomography, and when necessary lateral tomography, was repeated to estimate accurately the extent and nature of the residual disease. Very few patients were able to produce sputum; if this was available at least six specimens were examined and cultures set up. In the remaining cases a minimum of six (in some cases many more) laryngeal swabs were cultured. The swabs were taken singly on consecutive days for three days and the procedure repeated after an interval of a week.

The average period of observation in this country before thoracotomy was about six weeks.

\section{Radiological Assessment of Disease and its} Behaviour During Chemotherapy

Adopting the classification suggested by FosterCarter, Myers, Goddard, Young, and Benjamin (1952), the extent of the disease present initially was such that of the 59 cases, 29 were group I (one zone involved), 26 were group II (two or three zones), and four group III (four or more zones). There was radiological evidence of cavitation on admission in 39 patients $(66 \%)$. In these the presence of a total of 46 distinct cavities was confirmed tomographically.

Cavity size was assessed by measuring the maximum diameter on postero-anterior radiographs, or on tomographs if the cavities were not visualized on the simple radiograph. Cavities were recorded as small when the maximum diameter was $1.5 \mathrm{~cm}$. or less; large if it was greater than $2.5 \mathrm{~cm}$. ; and medium if between $1.5 \mathrm{~cm}$. and $2.5 \mathrm{~cm}$. There were 31 small cavities (67\% of the total), five medium cavities $(11 \%)$, and 10 large cavities (22\%).

\section{Cavity Closure}

Of the 46 cavities present at the beginning of treatment, the cavity closed, and this was confirmed tomographically in $41(89 \%)$.

It was found that at the end of three months' chemotherapy 18 cavities $(39 \%)$ had closed; at the end of six months $30(65 \%)$ had closed; and at the end of nine months $39(85 \%)$ of the original 46 cavities had closed (Table I). Two

TABLE I

TIME OF CLOSURE OF 46 CAVITIES IN 39 PATIENTS DURING PROLONGED CHEMOTHERAPY

\begin{tabular}{c|c}
\hline $\begin{array}{c}\text { Months } \\
\text { of Chemotherapy }\end{array}$ & $\begin{array}{c}\text { No. of Cavities Shown } \\
\text { to Have Closed }\end{array}$ \\
\hline 3 & $18(39 \%)$ \\
6 & $30(65 \%)$ \\
9 & $39(85 \%)$ \\
More than 9 & $41(89 \%)$ \\
\hline
\end{tabular}

cavities closed later than this, one at 12 months and the other at 13 months. The remaining five cavities persisted throughout treatment and were still present at operation (average time 16 months ; range 12-23 months); one of these cavities had closed after six months' treatment but reopened after a further four months and had not closed again before operation was undertaken two 
months later. This was the only cavity that did not remain closed once tomographic evidence of closure had been obtained. One case was not amenable to classification; a small rounded lesion was present in the posterior segment of the right upper lobe. This showed little change until 15 months' treatment had been completed, when the lesion appeared as a ring shadow $1 \mathrm{~cm}$. in diameter.

A relationship existed between initial cavity size and the length of time for which the cavity remained open, in that the greater the size of the cavity the longer was the period required to effect closure (Table II). The five cavities that

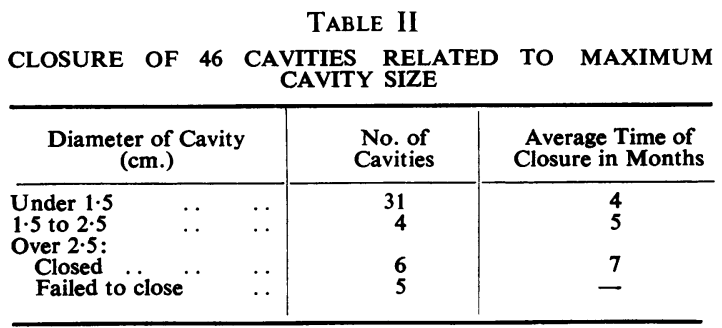

persisted until operation was undertaken were all large, the smallest measuring $2.5 \mathrm{~cm}$. in its maximum diameter on the admission radiograph.

\section{Radiological Regression During Treatment}

Radiological stability was judged to have been achieved when three consecutive routine films at six weeks' intervals had shown no change, and it was seldom necessary to modify this assessment after examination of the radiographs taken in the remaining period of observation. In eight patients $(13 \%)$ radiological change was still occurring at the time of operation despite the fact that they had received chemotherapy for an average of 17 months at that time (Table III) ; from only one of these were organisms subsequently grown from the resected specimen. Several cases of non-cavitated disease showed only slight radiographic change during treatment ; and in a few there was considerable change after

TABLE III

RADIOLOGICAL REGRESSION RELATED TO DURATION OF CHEMOTHERAPY

\begin{tabular}{|c|c|}
\hline Radiological Regression & No. \\
\hline $\begin{array}{l}\text { No change under chemotherapy } \\
\text { Radiological stability reached in } 6 \text { months or less } \\
\text { In } 6-12 \text { months } \\
\begin{array}{l}12-18 \\
\text { Radiological stability not }\end{array} \\
\text { operation .. } \\
\text { opeached at }\end{array}$ & $\begin{array}{l}2(3 \%) \\
14(23 \%) \\
24(42 \%) \\
11(19 \%) \\
8(13 \%)\end{array}$ \\
\hline
\end{tabular}

between six and 12 months' chemotherapy despit only slight change before this.

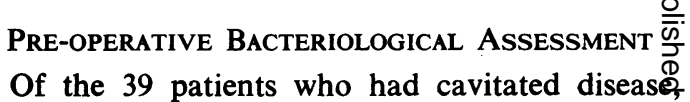
$20(52 \%)$ were reported to have tubercle bacilfi in the sputum on admission as compared witt only one $(5 \%)$ of 20 patients with no evidence of cavitation. Pre-treatment sensitivity tests were not carried out. In some cases, where there haid been contact with urban civilian populations the Far East, the possibility of infection with initially drug-resistant strains cannot be excluded but from the clinical behaviour of the cases it is considered unlikely.

In the whole series, from only two patients, both with persistent cavities, were organisms resistant to the drugs in use isolated at any stage. In one they were detected by laryngeal swab culture in the immediate pre-operative period. 道 the other they were first isolated after 11 month chemotherapy, and despite repeated negative sputum cultures over the succeeding 12 month, were again detected by laryngeal swab culture set up in the immediate pre-operative period.

These two cases illustrate the value of using laryngeal swab cultures in the assessment of cases showing unclosed cavities after long periods of chemotherapy. In both of these cases, and in no others, were resistant organisms subsequent类 recovered from the resected specimens.

\section{Surgical Aspects}

All operations were performed at the Connaught Hospital by one surgeon. Sixty thoracotomies were carried out in 59 patients. Five lobectomies, 49 resections of one or two segments, and six wedge resections wege performed. In some instances it was necessary to resect a wedge from another part of the lung 遮 those cases where lobectomy or segmential resection was the primary procedure. There we no complications that necessitated operative intervention, and none that prolonged convals cence by more than one or two weeks. In the operating theatre, the surgeon divided the resected lung segment or lobe ; one half was studied in the laboratory of the Connaught Hospital and the other was forwarded in a sterile container to the Institute of Diseases of the Chest, Brompton Hospital.

\section{EXAMINATION OF SPECIMENS}

Although the isolation of viable organisms from the operation specimen was the main objective of both laboratories, the work was carried 
independently and the procedures adopted consequently differed in several respects. As tissue removed at operation was divided between the two laboratories, in each of which separate specimens were taken for further examination, the total amount of material taken for culture was greater than would be commonly used, and it is possible that the chance of recovery of viable bacilli was thereby increased.

At the Connaught Hospital the purpose was to determine whether viable bacilli were still present in a given specimen rather than to determine the bacteriological state of particular lesions. Consequently the material from each specimen was pooled unless there was a lesion of considerable size which was taken alone. Films stained by the Ziehl-Neelsen method were prepared from each specimen and six culture media were inoculated. Each specimen was examined histologically.

At the Brompton Hospital an attempt was made to relate the bacteriological findings to the individual lesions. Clinical notes, a report of the operation findings, and radiographs were available at the time of examination of the specimen so that dissection could be performed to the greatest advantage. When a cavity was known to have been present particular care was taken to identify it or its residue. Several separate portions of diseased lung tissue (average three, maximum six), each usually representing an individual lesion, were taken from each specimen. One hundred and seventy-five lesions were thus taken, and two smear and culture examinations were made of each of these, one after treating with trisodium phosphate and the other of the untreated material, making a total of 350 samples. The method of handling the specimens and the details of bacteriological examination were similar to those described previously (Keers, Riddell, and Reid, 1956).

\section{ANALYSIS OF RESUlts}

Of the total of 350 samples (from 175 lesions) examined at the Institute of Diseases of the Chest 202 were smear positive, and 23 (from four patients) were culture positive. At the Connaught Hospital 60 samples (one from each operation case) were examined, of which 33 were smear positive and four culture positive. From only two specimens were positive cultures obtained in both laboratories; these were also the only two from which resistant strains were isolated, and came from the only two patients whose preoperative laryngeal swabs had been positive. In total, positive smears were obtained from specimens derived from 50 patients, but positive cultures were obtained from only six.

The duration of chemotherapy in the whole group ranged from 10 to 25 months, and the 59 patients were divided into four groups according to the duration of treatment they had received; the relation of the specimen culture results to these groups is shown in Table IV.

TABLE IV

\begin{tabular}{|c|c|c|}
\hline \multicolumn{3}{|c|}{$\begin{array}{l}\text { BACTERIOLOGY OF RESECTED LUNG SPECIMENS } \\
\text { RELATED TO DURATION OF CHEMOTHERAPY }\end{array}$} \\
\hline $\begin{array}{c}\text { Months } \\
\text { of } \\
\text { Chemotherapy }\end{array}$ & $\begin{array}{c}\text { No. of } \\
\text { Resected } \\
\text { Specimens }\end{array}$ & $\begin{array}{c}\text { No. of Cases with } \\
\text { Viable Bacilli } \\
\text { Recovered from Specimen }\end{array}$ \\
\hline $\begin{array}{l}9-12 \\
12-15 \\
15-18 \\
18-25 \text { (average 21) }\end{array}$ & $\begin{array}{r}5 \\
22 \\
19 \\
14\end{array}$ & $\begin{array}{l}1 \\
8 \\
2 \text { (one resistant) } \\
1(,,,, \quad)\end{array}$ \\
\hline
\end{tabular}

When the two cases with drug-resistant organisms are omitted (on the grounds that their chemotherapy was inadequate in these circumstances) the following results were obtained. From the resected specimens of five patients who had had 10 to 12 months' chemotherapy, bacilli were cultured from one $(20 \%)$. From 22 patients who had had 12 to 15 months' drug treatment, two specimens produced viable bacilli on culture $(9 \%)$. Eighteen patients received 15-18 months' chemotherapy, and the specimen from one $(5.5 \%)$ gave a positive culture, and of the specimens from 13 patients who were given more than 18 months' drug treatment, none grew organisms on culture. The numbers are too small to give a statistically significant result, but the downward trend in culture-positive results with increasing duration of chemotherapy is suggestive $(P=0.25)$. The same trend is noted when the number of culture-positive results obtained from the individual lesions and samples is taken into account (Table V).

The patients were divided into three groups according to the radiological features, so that further information might be obtained about the

TABLE V

BACTERIOLOGY OF LESIONS AND SAMPLES RELATED TO DURATION OF CHEMOTHERAPY (EXCLUDING THOSE WITH RESISTANT ORGANISMS)

\begin{tabular}{c|c|c|c|c}
\hline \multirow{2}{*}{$\begin{array}{c}\text { Months of } \\
\text { Chemotherapy }\end{array}$} & \multicolumn{2}{|c|}{ Lesions (222) } & \multicolumn{2}{c}{ Samples* (386) } \\
\cline { 2 - 4 } & $\begin{array}{c}\text { No. } \\
\text { Cultured }\end{array}$ & $\begin{array}{c}\text { No. } \\
\text { Positive }\end{array}$ & $\begin{array}{c}\text { No. } \\
\text { Cultured }\end{array}$ & $\begin{array}{c}\text { No. } \\
\text { Positive }\end{array}$ \\
\hline $9-12$ & 23 & $1(4.4 \%)$ & 41 & $2(4.9 \%)$ \\
$12-15$ & 86 & $4(4.8 \%)$ & 150 & $6(4 \%)$ \\
$15-18$ & 64 & $1(1.6 \%)$ & 110 & $1(0.9 \%)$ \\
More than 18 & 49 & 0 & 85 & 0
\end{tabular}

* The word " sample" describes a separate piece of tissue, of which several were sometimes taken from one lesion. 
influence of varying periods of chemotherapy on the bacteriology of specimens derived from patients with different types of disease. These groups were (A) those with cavities still present at the time of operation, (B) those in which cavities present initially had closed, and (C) those in which there had been no evidence of cavitation at any stage.

(A) Cavities Still Present at Time of OPERATION.-There were five such patients. Positive cultures were obtained from the specimens of three who had received 14, 16, and 23 months' chemotherapy respectively (Table VI). In addition to the five cases in which cavities had

TABLE VI

DURATION OF CHEMOTHERAPY RELATED TO SPECIMEN BACTERIOLOGY IN FIVE CASES WITH PERSISTENT CAVITATION

\begin{tabular}{c|c|c}
\hline $\begin{array}{c}\text { Months } \\
\text { of }\end{array}$ & $\begin{array}{c}\text { No. of } \\
\text { Resected } \\
\text { Specimens }\end{array}$ & $\begin{array}{c}\text { No. in which } \\
\text { Viable Bacilli were } \\
\text { Recovered from Specimen }\end{array}$ \\
\hline $\begin{array}{c}9-12 \\
12-15\end{array}$ & 1 & Nil \\
More than 18 & 2 & 1 \\
& 1 & 1 \\
\hline
\end{tabular}

persisted throughout treatment, a cavity was present in the operation specimen of one further case where a previously "solid" lesion cavitated just before operation and after 15 months' chemotherapy. All cultures from this specimen were negative.

Since the problem of persistent cavitation is important to the clinician the brief details of these cases are given. Cavity diameter is reported both as the maximum recorded at any period of treatment, which proved to be that assessed from the initial radiographs (except in Case 12), and as that recorded immediately before operation.

Case 4.-Cavity $2.5 \mathrm{~cm}$. ; under $1 \mathrm{~cm}$. The initial period of daily drug therapy was 30 days, and the total duration of chemotherapy before operation was 14 months. $M$. tuberculosis could not be grown from the cavity but was recovered from small nodules of caseous material in its vicinity. The organisms were fully sensitive to the drugs in use and to P.A.S.

Case 12.-Cavity $3.0 \mathrm{~cm}$; $3.0 \mathrm{~cm}$. The initial period of daily drug therapy was 90 days, and the total duration of chemotherapy 23 months. Numerous acid-fast bacilli were seen in smears from the specimen and confluent growths resulted on culture. From all lesions $M$. tuberculosis resistant to isoniazid, resistant or partially resistant to streptomycin, and sensitive to P.A.S. were obtained. The strains were sensitive to combinations of these drugs tested save isoniazid-streptomycin, to which partial resistance was demonstrated. The cavity wall showed evidence of active tuberculosis.
Case 16.-The initial period of daily drug therapy was 60 days and the total duration of chemotherapy was 12 months. A cavity $3.0 \mathrm{~cm}$. in diameter was present initially, but this was shown by tomography to be closed at six months; about four months later the cavity reopened and was still present $(1 \mathrm{~cm}$. in diameter) at operation. Acid-fast bacilli were seen on some smears but all cultures proved negative. Histologically, the cavity wall still showed evidence of tuberculous activity.

Case 22.-Cavity $3 \mathrm{~cm} . ; 1 \mathrm{~cm}$. The initial period of drug therapy was 60 days, and the total duration of chemotherapy 17 months. Very few bacilli were $\vec{A}$ seen in direct smears, but heavy growths of iv $M$. tuberculosis were obtained from 18 of the $20 \vec{\circ}$ cultures set up. The organisms were resistant to $₫$ streptomycin in all instances. All cultures proved 옥 sensitive to other drugs, and to combinations of streptomycin and other drugs, save three in which $\breve{c}$ partial resistance to isoniazid alone was evident. The cavity was formed by caseous material with many giant cells and epithelioid cells and tubercles $\vec{\theta}$ were numerous in the walls of the draining bronchus.

Case 27.-Cavity $5.0 \mathrm{~cm}$; $5.0 \mathrm{~cm}$. The initial period of daily drug therapy was 90 days, and the total duration of chemotherapy was 14 months. From none of the specimens taken from the cavity and the neighbouring small nodules were smears $\stackrel{\mathbb{D}}{\circ}$ positive and all cultures were negative. No bronchial communication with the cavity could be demonstrated. Histologically the cavity wall was "healing," but by strict criteria it could not be considered $\supset$ healed. It consisted of dense fibrous tissue with some giant cells, epithelioid cells, and hyalinized caseous material.

(B) Disease with Cavities Closed before $\stackrel{0}{x}$ OPERATION.-There were 34 patients in whom all $\dot{5}$ cavities had closed before operation. Positive cultures were obtained from the specimens $\delta$ derived from these patients in only one instance. The total duration of chemotherapy received by 음 these patients before operation is shown in $D$ Table VII.

\section{TABLE VII}

DURATION OF CHEMOTHERAPY RELATED TO SPECIMEN BACTERIOLOGY IN 34 CASES WITH SOLID FOCI RESIDUAL

\begin{tabular}{c|c|c}
\multicolumn{2}{|c|}{ FROM PREVIOUS CAVITIES } \\
\hline $\begin{array}{c}\text { Months } \\
\text { of } \\
\text { Chemotherapy }\end{array}$ & $\begin{array}{c}\text { No. of } \\
\text { Resected } \\
\text { Specimens }\end{array}$ & $\begin{array}{c}\text { No. in which } \\
\text { Viable Bacilli } \\
\text { Recovered from Specimen }\end{array}$ \\
\hline $9-12$ & 2 & 1 \\
$12-15$ & 12 & 0 \\
$15-18$ & 10 & 0 \\
More than 18 & 10 & 0 \\
\hline
\end{tabular}

The one patient who gave a positive culture $\stackrel{\mathbb{Q}}{\mathscr{Q}}$ from the resected specimens was found to be the $\overline{0}$ only one in the group who had received drugs for 8 less than six months after cavity closure. $\mathrm{He}$ 
also had received chemotherapy for less than one year altogether. In this case smears were negative and $M$. tuberculosis was not recovered by culture from the lesion representing the cavity, but from some separate small nodules in an adjacent lung segment. Histologically these nodules showed characteristic tuberculous granulation tissue in their wall, but calcification had begun as shown by basophilia of the caseous material. The organisms cultured were fully sensitive to the drugs in use and to P.A.S.

In this group care was taken to determine the fate of the cavities which were known to have been present initially. In the 34 specimens, a " filled-in" cavity was found in 15 , a nodule of caseous material in 12, in two thickened pleura was the only residue, whilst in five specimens it was not possible to be certain which lesion represented the previous cavity. In four cases two cavities had been recognized radiologically, but the residue of only one cavity was found in the specimen. The remaining three cavities noted to be present in the series and to have closed under treatment (see Table I) represented contralateral disease which was not resected.

The "filled-in" cavities include scars which contained only a small amount of caseous material, only 1 or $2 \mathrm{~mm}$. in diameter, as well as those in which a larger collection of caseous material was included within a thickened irregular wall. The "nodules of caseous material" are recorded separately, since their size varied between 1 and $3 \mathrm{~cm}$. and was often greater than the "filled-in cavities." More characteristically, their capsules were thin with little distortion of surrounding lung, making them indistinguishable from many nodules of caseous pneumonia in which cavitation had never been recorded. In these specimens the lesion representing the cavity was identified in the light of examination of the radiographs and tomograms.

(C) Specimens from Patients with Solid Lesions Throughout.-Positive cultures were obtained from the specimens of two of the 20 patients in this group (Table VIII).

TABLE VIII

DURATION OF CHEMOTHERAPY RELATED TO SPECIMEN BACTERIOLOGY IN 20 SPECIMENS OF SOLID DISEASE NEVER CAVITATED

\begin{tabular}{c|c|c}
\hline $\begin{array}{c}\text { Months } \\
\text { of } \\
\text { Chemotherapy }\end{array}$ & $\begin{array}{c}\text { No. of } \\
\text { Resected } \\
\text { Specimens }\end{array}$ & $\begin{array}{c}\text { No. in which } \\
\text { Viable Bacilli } \\
\text { Recovered from Specimen }\end{array}$ \\
\hline $9-12$ & 2 & 0 \\
$12-15$ & 8 & 1 \\
$15-18$ & 7 & 1 \\
More than 18 & 3 & 0 \\
\hline
\end{tabular}

Case 18.-The initial period of daily drug therapy was 30 days, and the total duration of chemotherapy 14 months. The disease was discovered on routine radiological examination and at no time during treatment was a positive sputum or laryngeal swab reported. There were two small rounded shadows $1 \mathrm{~cm}$. in diameter in the apical segment of the right upper lobe which showed little radiological change during the first five months of treatment and none thereafter. Five samples from three lesions in the specimen were smear positive, but from only one were cultures positive. Since subculture failed drug sensitivities could not be determined. Histological examination of the lesion from which the positive culture was obtained showed areas of caseation surrounded by endothelial cells and lymphocytes, and in some areas by dense fibrous tissue. There was some evidence of early calcification.

Case 36.-The initial period of daily drug therapy was 30 days, and the total duration of chemotherapy was 16 months. A circumscribed shadow $2 \mathrm{~cm}$. in diameter in the apical part of the left upper lobe was discovered on routine radiological examination. A calcified primary complex was present on the right side. Sputum and laryngeal swabs were negative throughout. The shadow became slightly smaller during treatment, but in the fifteenth month of treatment (just before operation) a small shadow appeared in the right lower zone. This disappeared during the next few months, although chemotherapy remained unchanged throughout. The lesion in the left upper lobe was resected and two of the three samples from the specimen were smear positive, and from one $M$. tuberculosis was cultured. Drug sensitivities could not be determined because subculture failed. The main lesion was a collection of caseous material $2 \mathrm{~cm}$. in diameter with several worm-like extensions from it. Histological examination of part of the large lesion showed it mostly enclosed in dense fibrous tissue, but on one side poorly defined by tuberculous granulation tissue spreading into the lung.

\section{Discussion}

There is difference of opinion concerning the place of resectional surgery in the treatment of pulmonary tuberculosis (Lancet, 1958). The present trend is to advise surgery less often (Wier, 1956), and to place greater reliance on long-term chemotherapy (Crofton, 1955; Ross, Horne, Grant, and Crofton, 1958), save in special circumstances such as the detection of resistance (Stewart and others, 1956) or the persistence of cavitation (Hoyle, Nicholson, and Dawson, 1955 ; Ware, Stauss, Dillon, and Tempel, 1956). The logical outcome of this trend may well be to reserve surgery only for the failures of treatment by chemotherapy. Bottrill and Edge (1957) rightly state that the ultimate reliability of 
continued chemotherapy alone can only be assessed by long-term clinical follow-up, but this will involve waiting some years. Until that time bacteriological study of resected specimens is likely to provide the best guidance in such problems as the efficacy of particular drug régimes, the duration for which they should be continued, and the type of disease for which surgery should still be advised.

Nevertheless the results of culture studies of resected tuberculous lesions should take into account the number of lesions examined from any one patient, since selection of material from a number of sites will favour a higher yield of positive cultures. For example, from one patient in this series in whom a cavity had been present but had closed during treatment, organisms were not grown from the lesion representing the cavity but were cultured from some separate small nodules in an adjacent segment.

The methods of study that we have adopted bear closer comparison with those of Bottrill and Edge (1957), in which the material was collected from all major lesions, than with those of Cotter and others (1958), who examined only individual lesions. The overall results reported by Bottrill and Edge (1957) from a study of a group of 54 patients treated with "satisfactory" chemotherapy were similar to those in the present series in which the régime consisted of streptomycin and isoniazid on alternate days for long periods after a short initial period of daily drugs.

The administration of streptomycin and isoniazid together on alternate days, following an initial period of daily therapy, is a less common practice than that of giving streptomycin on alternate days and isoniazid daily. The former régime has been used by Mackay-Dick and Rothnie (1954), Snelling (1957), Todd and others (1956), and Mackay-Dick and Slattery (1958). Hutton, Lutalo, Williams, Tonkin, and Fox (1956) used alternate-day chemotherapy after a 12-week period of daily streptomycin and isoniazid as one of their drug régimes in the treatment of acute bilateral pulmonary tuberculosis in East Africans; they found it was effective when assessed after six months' treatment. In our series, judged by sputum conversion, incidence of resistance, cavity closure, radiological regression of lesions, and the bacteriological state of the resected specimens, the results of our régime of chemotherapy appear good.

Another consideration is a commonly held belief that the Gurkha is particularly susceptible to tuberculosis, and that when the disease occurs it is likely to be acute and rapidly progressive. In $\stackrel{\overrightarrow{\vec{\rho}}}{\vec{f}}$ this series no evidence was found to support thiso view; in addition the clinical and radiologicalo response to treatment did not appear to differ from $\frac{\bar{c}}{5}$ that seen in European patients with comparable $\mathbb{\Phi}_{\Omega}^{\mathbb{\Phi}}$ disease. Similar observations were made byo Robinson (1956).

The information gained from a bacteriological $\vec{\circ}$ study of resected specimens is enhanced by $\vec{\omega}$ correlation with the clinical and radiologicalo findings. The patients in this study have, there $-\overrightarrow{\vec{x}}$ fore, been divided into the three groups already detailed. The uniformity of chemotherapy given in this series still further enhances the value of $\vec{A}$ such a correlation.

(A) Persistent Cavitation.-Five patients haf cavities that persisted through treatment. Two of these had positive pre-operative laryngeal swab cultures, and both proved resistant to the drugs in use. The specimens in both these cases produced positive cultures, which also were resistant. In the remaining three cases laryngeal swabs were repeatedly negative on culture, but a positive culture was obtained from one of the threes resected specimens, although this was not from the cavity itself.

Most authorities would agree that resection is indicated in patients with positive laryngeal swabo cultures, evidence of resistance to two of the main? antibacterial drugs, and persistent cavitation afte periods of treatment of the order given to these. patients. In a follow-up assessment of the results of continued chemotherapy (average duration 1 次 months) in the treatment of pulmonary tuberculosis, Raleigh (1957) found that withoug surgery the prognosis of patients with persistent cavitation and persistent positive sputum waş poor; this group accounted for most of the relapses and all of the deaths from tuberculosis in his large series. Furthermore, a close association between persistent cavities and the recovery of resistant organisms from the specimens is recognized (Stewart and others, 1956), and, in our series, the only drug resistance encountered was in patients with persistent cavitation.

In those with persistent cavitation and negative sputum or laryngeal swab cultures there is lesf agreement concerning management. Of three such patients in this series, one still harboured viable bacilli, but there was no clinical of radiological feature which distinguished this cas $\Phi$ from the other two. Although the possibilit remains that complete epithelialization of such cavities can occur, no clinical investigations car prove that this has indeed happened, and it ha\& 
been established that cavities apparently healed on clinical assessment are often found not to be so on histological and bacteriological examination of the specimen (Keers and others, 1956). A study of "open negative" patients reported by Bell, Decker, and Raleigh (1957) showed that the relapse rate in those treated by chemotherapy alone was much higher than in those whose treatment included resection.

These findings constitute a strong argument for advising resection in such cases. Since the chance of subsequent closure of cavities that persist after nine months' chemotherapy is so small we consider that this would be the logical time at $v$. hich to reassess the case and consider resection.

(B) Cavities Closed under Treatment.-Of cavities present initially, $85 \%$ had closed before nine months' treatment had been completed (Table I), and the percentage closure at seven months was almost 80 . This finding corresponds closely with that of Ross and Kay (1956), who calculated that $79 \%$ closure was to be expected following treatment with daily drugs for eight months. Cotter and others (1958) reported a closure rate of $80 \%$, but considered that the strict postural retention, which had been carried out in most of their cases, played a significant role in achieving this. This practice was not adopted in the treatment of the patients we describe.

In most studies of resected specimens no distinction has been made between those solid lesions which were previously cavitated and those which were solid throughout, although cavitation at any time in the history of a lesion has often been taken as an indication for resection (Jones, 1953 ; Keers, 1953 ; Ware and others, 1956).

In those cases in this series in which cavity closure has been achieved, and sputum or laryngeal swab cultures have remained negative, viable bacilli have not persisted in the residual lesion provided chemotherapy had been continued for more than six months after cavities had closed, and the total duration of chemotherapy had exceeded one year. If chemotherapy were undertaken as definitive treatment in such cases the current practice would be to continue drugs for one year after cavity closure (Ross and others, 1958). From the results in this series it appears that in this type of disease, chemotherapy for this period of time is really effective treatment, and that previous cavitation in itself is not a strong indication for resection. Long-term follow-up of such cases will provide the final proof of the efficacy and the reliability of chemotherapy alone. Until that time the clinician has no alternative save to weigh the evidence provided by studies such as those reported above against the known, but small, mortality and morbidity rate of resection.

(C) Non-Cavitated Disease.-There were only 20 cases in this group; the main facts relating to them are summarized in Table VII. In two patients the specimens proved culture positive; one had received 14 and the other 16 months' chemotherapy. Both cases had been consistently sputum and laryngeal swab negative, they had only small lesions, and they had shown but slight radiological change. The patient who had been treated for 16 months also developed a new lesion in the opposite lung just before surgery was undertaken.

The danger of leaving such minimal lesions untreated has often been stressed. In patients of the age group with which we are dealing, Springett (1956) reported evidence of activity within five years in from $10 \%$ to $50 \%$ of cases, depending on the initial size of the lesion. Of minimal lesions considered active and treated with chemotherapy, Todd (1958) quotes a series followed up by the Royal Air Force, in which the relapse rate was $7.7 \%$ over three years. These patients were given only about six months' chemotherapy, and we know of no similar followup of patients treated for longer periods for similar disease. In 1956 Todd and others reported a series of 238 such cases in which resection was undertaken. In 105 the specimen proved culture positive, and surgery after a short period of chemotherapy was advocated.

In the present state of knowledge, the finding of viable bacilli after 16 months' chemotherapy favours caution in the management of such disease. It is probably for this type of disease that clinicians now advise resection least often; furthermore, the duration of chemotherapy advised by many is probably often considerably less than that used for more extensive disease. We hesitate to advocate surgery as a routine procedure for disease of this nature, but we think it still merits serious consideration, particularly in special circumstances of the kind existing with the patients described in this study. Even when adequate facilities for continued supervision and long-term chemotherapy exist there may still be additional factors which influence the decision to advise resection, as in the case of the professional soldier who wishes to continue his career and for whom the best possible guarantee of future fitness under arduous conditions is needed (Large, Curry, and Harrison, 1957 ; Mackay-Dick, 1958). 
It is unlikely that many further reports of specimen studies following prolonged chemotherapy for such disease will be possible, since under normal circumstances in civilian practice resection will either be undertaken early (Todd and others, 1956) or not at all ; we feel, therefore, that the problem of the management of noncavitated disease still merits attention.

\section{SUMMARY}

Fifty-nine Gurkha soldiers suffering from pulmonary tuberculosis had partial lung resection performed after treatment with the same régime of chemotherapy for periods between 10 and 25 months. This consisted of $1.0 \mathrm{~g}$. streptomycin and $100 \mathrm{mg}$. b.d. isoniazid together on alternate days after a short period of daily drug therapy.

Radiological assessment included full-plate tomography and routine postero-anterior chest radiographs at six-week intervals in every case. That cavities had closed, when this was so, was confirmed tomographically.

Laryngeal swab culture was carried out in the pre-operative period in every case and was positive in two patients only, whose specimens were the only two yielding resistant bacilli.

The patients were divided into three groups according to the type of their disease, and their clinical and radiological features are described. These are correlated with the findings of bacteriological study of the resected residual disease.

In general, the findings support the view that the persistence of viable tubercle bacilli in the lesions is reduced with increased duration of chemotherapy, unless bacterial resistance to the drugs used in treatment has developed.

There were five patients in whom cavities had persisted throughout treatment. The lesions from three of these were culture positive, the organisms in two being resistant to streptomycin and isoniazid.

Of 34 instances in which the solid residuum of previously cavitated disease was resected, tubercle bacilli were cultured from the specimen in only one, and then not from the cavity site itself. This patient had received only four months' chemotherapy after the cavity had closed, whereas 30 of the remaining 32 patients had received from six to more than 18 months' such chemotherapy. The possible implications of this finding are discussed.

Of 20 patients with non-cavitated disease throughout, tubercle bacilli were cultured from the resected specimens in two ; these patients had $\frac{\text { ? }}{0}$ received chemotherapy for 14 and 16 months $\frac{C}{O}$ respectively. Both cases had been consistently $\frac{\bar{\sigma}}{0}$ sputum negative, had negative pre-operative $\frac{\widetilde{\sigma}}{\widetilde{\sigma}}$ laryngeal swabs, and had shown only slight $\varrho$ radiological change during treatment. It is suggested that minimal lesions of this type may $\vec{\circ}$ well prove to pose a more difficult problem with regard to their definitive treatment than has beenc thought.

We should like to thank Sir Geoffrey Todd, $\overrightarrow{+}$ K.C.V.O., O.B.E., for his interest and advice. Weiv acknowledge with gratitude the co-operation of $\mathrm{Dr} \cdot \overrightarrow{\mathrm{H}}$ J. D. Laycock, anaesthetist to the Connaught Hospital, Captain M. F. R. Waters, R.A.M.C.은 (1955-6), Captain D. A. D. Slattery, M.B.E., R.A.M.C. (1956-8), who looked after the patients in Malaya, ande Captains J. R. Rawstron and D. J. Pollock, R.A.M.C., who were responsible for the laboratory work at the ${ }^{\mathbb{D}}$ Connaught Hospital. We should also like to thank $\vec{e}$ the technical staffs of the Bacteriology Department, $\mathcal{O}$ Brompton Hospital, and of the Morbid Anatomy Department, Institute of Diseases of the Chest.

\section{REFERENCES}

Auerbach, O., Hobby, G. L., Small, M. J., Lenert, T. F., and Comer J. V. (1955). J. thorac. Surg., 29, 109.

Bell, J. W., Decker, A. M., and Raleigh, J. W. (1957). Amer. Rev $\overrightarrow{\bar{\sigma}}$ Tuberc., 75, 538 .

Bottrill, D. E., and Edge, J. R. (1957). Tubercle (Lond.), 38, 303.

Cotter, D. J., Foreman, H. M., and Seal, R. M. E. (1958). Thorax, $13,150$.

Crofton, J. W. (1955). Brit. med. J., 1, 1526.

d'Esopo, N., Bernstein, S., Decker, A. M., Raleigh, J. W., and

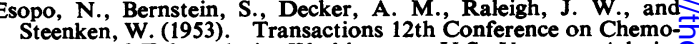
therapy of Tuberculosis, Washington; U.S. Veterans Administration, p. 229

Foster-Carter, A. F., Myers, M., Goddard, D. L. H., Young, F. H. \} and Benjamin, B. (1952). Brompton Hosp. Rep., 21, 1.

Hoyle, C., Nicholson, H., and Dawson, J. (1955). Lancet, 2, 1310.

Hurford, J. V., and Valentine, W. H. (1957). Tubercle (Lond.), 38, 194.

Hutton, P. W., Lutalo, Y. K., Williams, A. W., Tonkin, I. M., and Fox, W. (1956). Ibid., 37, 151.

Jones, J. C. (1953). Panel discussion, Amer. Rev. Tuberc., 68, 830.

Keers, R. Y. (1953). Edinb. med. J., 60, 128.

Riddell, R. W., and Reid, L. (1956). Tubercle (Lond.), 37, 404

Lancet (1958). 1, 1373 [Leading Article].

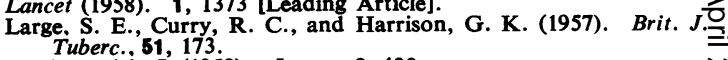

Mackay-Dick, J. (1958). Lancet, 2, 422

- and Rothnie, N. J. (1954). Tubercle (Lond.), 35, 182.

and Slattery, D. (1958). Brit. med. J., 1, 888.

Raleigh, J. W. (1957). Amer. Rev. Tuberc., 76, 540.

Robinson, T. M. (1956). Proc. roy. Soc. Med., 49, 324.

Ross, J. D., and Kay, D. T. (1956). Thorax, 11, 1.

Horne, N. W., Grant, I. W. B., and Crofton, J. W. (1958) Brit. med. J., 1, 237.

Snelling, M. R. J. (1957). Transactions of the First pan-Malayare Tuberculosis Conference. Singapore, 1956, p. 6.

Springett, V. H. (1956). Minimal Pulmonary Tuberculosis Found by Mass Radiography; A report to the Prophit Committee of the Mass Radiography; A report to the Prophit Committ
Royal College of Physicians. H. K. Lewis, London.

Stewart, S. M., Turnbull, F. W. A., and Macgregor, A. R. (1956). Tubercle (Lond.), 37, 388.

Todd, G. (1958). Personal communication.

Ware, P. F., Stauss, H. K., Dillon, R. J., and Tempel, C. W. (1956) Amer. Rev. Tuberc., 73, 165.

Wier, J. A. (1956). J. Amer. med. Ass., 162, 471. 